\title{
The Exponential Attractor for a Class of Nonlinear Coupled Kirchhoff Equations with Strong Linear Damping
}

\author{
Guoguang $\operatorname{Lin}^{1} \&$ Lingjuan $\mathrm{Hu}^{2}$ \\ ${ }^{1,2}$ School of Mathematics and Statistics, Yunnan University, Kunming, China \\ Correspondence: School of Mathematics and Statistics, Yunnan University, Kunming, China. E-mail: gglin@ynu.edu.cn \\ \&879694199@qq.com
}

Received: May 31, 2018 Accepted: June 15, 2018 Online Published: August 9, 2018

doi:10.5539/jmr.v10n5p49

URL: https://doi.org/10.5539/jmr.v10n5p49

\begin{abstract}
This paper investigates the dynamics for a class of nonlinear higher-order coupled Kirchhoff equations with strong linear damping. By means of the method proposed by Eden et al., the Lipschitz continuity and the discrete squeezing property of its solution semigroup are proved, and thus the existence of the exponential attractor is obtained.
\end{abstract}

Keywords: higher-order Kirchhoff-type, Lipschitz continuity, discrete squeezing property, exponential attractor

\section{Introduction}

In 1990, an exponential attractor for a continuous map $S$ which conducts on a compact invariant set $B$ was defined by Eden et al. As an inertial set, and as a compact, invariant finite dimensional subset $M$ of $B$ containing the global attractor $A$, which is the $\omega$-limit set of $B$, and all points of $B$ are attracted at least exponential rate. The recently developed exponential attractor theory retains many aspects of the global attractor and inertial manifold. The exponential attractor and global attractor, the main difference is that, once they are in an absorbing ball, all the solutions converge to the exponential attractor in an exponential rate, so the exponential attractor contains the global attractor, and the stable manifold convergence is only polynomial; but comparing to the inertial manifold, it also has finite dimension and attracts the solution exponentially, while the exponential attractor is not needed to have a manifold structure. The simple constructive way for exponential attractor is to restrict the inertial manifold to an absorbing set. But anyway, in general, when all the sets exist, they have the following relationship: $A \subseteq M \subseteq \mu \bigcap B \subseteq B$, set $M=\mu \bigcap B, M$ is an exponential attractor.

Initially, we recall the exponential attractors of some equations that have been certified.

In cooperation with Eden, (Milani, 1992) obtained some conclusions on the existence of exponential attractors for the semi-linear damped wave equation, especially considering the case of nonlinear term in three-dimensional space:

$$
u_{t t}+u_{t}-\Delta u+g(u)=f .
$$

Next, (Brochet et al., 1994) considered the system of equations with simultaneous order-disordered and phase separation dynamics in $N \leq 3$, the existences of the inertial set and the maximum attractor were proved and the upper bound of the fractal dimension of the attractor was obtained. Subsequently, the existence of exponential attractors was established by (Eden \& Rakotoson, 1994), that is, there is a sufficient condition for DSP to guarantee its existence. (Eden \& Kalantarov, 1996) simplified the framework by introducing a unified method to both the existence of exponential attractor by $\alpha$ contraction and the construction of exponential attractor by some Lipschitizianity condition of nonlinear operator. (Eden et al., 1998) had an improvement in the original construction of exponential attractor.

In recent years, an exponential attractor for second-order lattice dynamical system with nonlinear damping was constructed by (Fan \& Yang, 2010). Secondly, the strongly damped wave equation: $u_{t t}-\Delta u_{t}-\Delta u+\varphi(u)=f(x)$, its exponential attractor was studied in (Yang \& Sun, 2010; Li \& Yang, 2013). By using a method based on weak quasi-stability estimation, the existence of exponential attractor for the Kirchhoff equation with strong nonlinear damping and supercritical nonlinearity was proved by (Yang \& Liu, 2015):

$$
u_{t t}-\sigma\left(\|\nabla u\|^{2}\right) \Delta u_{t}-\phi\left(\|\nabla u\|^{2}\right) \Delta u+f(u)=h(x)
$$

Ma, Q. Z and her students considered the existence of exponential attractor with method proposed by Eden et al. Through 
non-classical diffusion equation, Kuramoto-Sivashinsky equation, nonlinear beam equation, dissipative MKdV equation, nonlinear reaction-diffusion equation with derivative term, drawbridge equation, and nonlinear stretchable beam equation, see (Ma \& Liu, 2011; Gao \& Ma, 2011; Wang et al., 2011; Han \& Ma, 2011; Kang \& Ma, 2012; Wang \& Ma, 2016; Jia \& Ma, 2017).

Authors in (Shang \& Guo, 2005) considered the global fast dynamics of the generalized symmetric regularized long wave equation with damping term and got the squeezing property of nonlinear semigroup and the existence of the exponential attractor. (Lin et al., 2017) studied the global dynamics of a nonlinear generalized Kirchhoff-Boussinesq equation with damping term and proved the existence of its exponential attractor:

$$
u_{t t}+\alpha u_{t}-\beta \Delta u_{t}+\Delta^{2} u=\operatorname{div}\left(g\left(|\nabla u|^{2}\right) \nabla u\right)+\Delta h(u)+f(x) .
$$

The exponential attractors of higher-order nonlinear Kirchhoff equation were analyzed by (Chen et al., 2016):

$$
u_{t t}+(-\Delta)^{m} u_{t}+\phi\left(\left\|\nabla^{m} u\right\|^{2}\right)(-\Delta)^{m} u+g(u)=f(x)
$$

Inspired by the above, this article arranges as follows. In Part 2, some of the main preliminaries are stated, and in Part 3, the Lipschitz continuity and discrete squeezing property of semigroup are acquired, thereby exponential attractor is established.

$$
\begin{gathered}
u_{t t}+M\left(\left\|D^{m} u\right\|^{2}+\left\|D^{m} v\right\|^{2}\right)(-\Delta)^{m} u+\beta(-\Delta)^{m} u_{t}+g_{1}(u, v)=f_{1}(x), \quad \text { in } \Omega \times[0,+\infty), \\
v_{t t}+M\left(\left\|D^{m} u\right\|^{2}+\left\|D^{m} v\right\|^{2}\right)(-\Delta)^{m} v+\beta(-\Delta)^{m} v_{t}+g_{2}(u, v)=f_{2}(x), \quad \text { in } \Omega \times[0,+\infty), \\
u(x, 0)=u_{0}(x), \quad u_{t}(x, 0)=u_{1}(x), \quad x \in \Omega, \\
v(x, 0)=v_{0}(x), \quad v_{t}(x, 0)=v_{1}(x), \quad x \in \Omega, \\
\frac{\partial^{i} u}{\partial n^{i}}=0, \quad \frac{\partial^{i} v}{\partial n^{i}}=0, \quad i=0,1,2, \cdots, m-1, \quad x \in \partial \Omega, t \geq 0,
\end{gathered}
$$

where $\Omega$ is a bounded domain in $R^{n}$ with smooth boundary $\partial \Omega, \beta>0$ is real number and $m \geq 1$ is positive integer,

$M(s)$ is a nonnegative $C^{1}$ function, $g_{j}(u, v)$ and $f_{j}(x)(j=1,2)$ are nonlinear terms and external force terms respectively.

\section{Preliminaries}

For convenience, we need the following notations in subsequent article. Considering a family of Hilbert spaces $V_{\alpha}=D\left(A^{\alpha / 2}\right), \alpha \in R$, whose inner product and norm are given by $(\cdot, \cdot)_{V_{\alpha}}=\left(A^{\alpha / 2}, A^{\alpha / 2}\right)$ and $\|\cdot\|_{V_{\alpha}}=\left|A^{\alpha / 2} \cdot\right| \cdot$ Apparently

$$
\begin{gathered}
H=V_{0}=L^{2}(\Omega), \quad V_{m}=H^{m}(\Omega) \bigcap H_{0}^{1}(\Omega), \quad V_{2 m}=H^{2 m}(\Omega) \bigcap H_{0}^{1}(\Omega), \\
E_{0}=V_{m} \times H \times V_{m} \times H, \quad E_{1}=V_{2 m} \times V_{m} \times V_{2 m} \times V_{m} .
\end{gathered}
$$


We make the following hypotheses:

(H1) $M(s) \in C^{1}\left(R^{+}\right)$and for positive constants $m_{0}, m_{1}$,

(1) $0<m_{0} \leq M(s) \leq m_{1}$

(2) $m^{*}=\left\{\begin{array}{l}m_{0}, \frac{d}{d t}\left(\left\|D^{m} u\right\|^{2}+\left\|D^{m} v\right\|^{2}\right)>0 . \\ m_{1}, \frac{d}{d t}\left(\left\|D^{m} u\right\|^{2}+\left\|D^{m} v\right\|^{2}\right)<0 .\end{array}\right.$

(H2) $g_{j}(u, v)(j=1,2) \in C^{1}(R)$ such that

$$
\begin{cases}\left|g_{1 u}(u, v)\right| \leq C\left(1+|u|^{r-1}+|v|^{r}\right), & \left|g_{1 v}(u, v)\right| \leq C\left(1+|u|^{r}+|v|^{r-1}\right) . \\ \left|g_{2 u}(u, v)\right| \leq C\left(1+|u|^{r^{\prime}-1}+|v|^{r^{\prime}}\right), & \left|g_{2 v}(u, v)\right| \leq C\left(1+|u|^{r^{\prime}}+|v|^{r^{r^{-1}}}\right) .\end{cases}
$$

Where $2 \leq r\left(r^{\prime}\right) \leq \frac{2 n}{n-2 m}$.

Definition 1 (Eden et al., 1994) A compact set $M$ is called an exponential attractor for $\{S(t), B\}$ if

1) $A \subseteq M \subseteq B$, where $A$ is the global attractor;

2) $S(t) M \subseteq M$, for all $t \geq 0$, that is, $M$ is positively invariant under $S(t)$;

3) $M$ has finite fractal dimension;

4) There exist universal constants $c_{1}, c_{2}$, such that for every $u \in B$,for every natural number $t$, $\operatorname{dist}(S(t) u, M) \leq c_{1} e^{-c_{2} t}$.

Definition 2 (Eden et al., 1994) A solution semigroup $\{S(t)\}_{t \geq 0}$ is said to satisfy the discrete squeezing property (DSP) if there exists $t_{*}>0$ such that the map $S_{*}=S\left(t_{*}\right)$ satisfies: there exists an orthogonal projection $P$ of finite rank $N$ such that, for every $u$ and $v$ in $B$, either

$$
\left\|Q_{N}\left(S_{*} u-S_{*} v\right)\right\|_{X} \leq\left\|P_{N}\left(S_{*} u-S_{*} v\right)\right\|_{X},
$$

or

$$
\left\|S_{*} u-S_{*} v\right\|_{X} \leq \frac{1}{8}\|u-v\|_{X}
$$

Where $Q_{N}=I-P_{N}$.

Definition 3 (Eden et al., 1994) We say $S(t)$ is Lipschitz continuous in the compact set $B$, if there exists a local bounded function $L(t)$ such that

$$
\|S(t) x-S(t) y\|_{X} \leq L(t)\|x-y\|_{X}
$$

for all $x, y \in B$. Here $L(t)$ does not depend on $x$ and $y$.

Theorem 1 (Eden et al., 1994) If the solution semigroup $\{S(t)\}_{t \geq 0}$ satisfies the discrete squeezing property on $B$ and if the map $S_{*}=S\left(t_{*}\right)$ is Lipschitz with Lipschitz constant $L$, then there exists an exponential attractor $M$ for the solution semigroup satisfying

$$
d_{F}(M) \leq N_{0}\left\{1, \frac{\ln (16 L+1)}{\ln 2}\right\}
$$




\section{The Existence of Exponential Attractor}

In this section, we prove equations (1.1)-(1.2) admit an exponential attractor, we verify the Lipschitz continuity and the discrete squeezing property of the dynamical system $S(t)$ in $E_{0}$.

First, we introduce $A=-\Delta$, since $A$ is self-adjoint, positive operator and has a compact inverse. Let $\left\{\lambda_{i}\right\}_{i=1}^{\infty}$ be the sequence of the eigenvalues and $\left\{\omega_{i}\right\}_{i=1}^{\infty}$ the corresponding sequence of eigenvectors,

$$
A \omega_{i}=\lambda_{i} \omega_{i} \quad\left(0<\lambda_{1} \leq \lambda_{2} \leq \cdots \leq \lambda_{i} \leq \cdots \rightarrow+\infty, \quad i \rightarrow+\infty\right) .
$$

Set $H_{N}=\operatorname{span}\left\{\omega_{1}, \omega_{2}, \cdots, \omega_{N}\right\}, \quad p_{N}$ is the orthogonal projection onto $H_{N}$ and $q_{N}$ is the orthogonal projection onto the orthogonal complement $H_{N}$, that is

$$
p_{N}: H \rightarrow H_{N}, \quad q_{N}=I-p_{N},
$$

and by the definition of projection

$$
\left|A^{m / 2} u\right| \geq \lambda_{N+1}^{m / 2}|u|, \quad\left|A^{m / 2} v\right| \geq \lambda_{N+1}^{m / 2}|v|, \quad \forall(u, v) \in q_{N} H \times q_{N} H .
$$

Make

$$
P_{N}: E_{0} \rightarrow\left(p_{N} V_{m}\right) \times\left(p_{N} H\right) \times\left(p_{N} V_{m}\right) \times\left(p_{N} H\right), \quad Q_{N}=I-P_{N},
$$

then

$$
P_{N}(u, p, v, q)=\left(p_{N} u, p_{N} p, p_{N} v, p_{N} q\right), \quad z=(u, p, v, q) \in E_{0} .
$$

For each $z=(u, p, v, q) \in E_{0}, p=u_{t}, q=v_{t}$, we construct functions as following

$$
\begin{gathered}
G(z)=m^{*}\left(\left|A^{m / 2} u\right|^{2}+\left|A^{m / 2} v\right|^{2}\right)+\left(|p|^{2}+|q|^{2}\right), \\
F(z)=\left(m^{*}+\frac{\beta}{2}\right)\left(\left|A^{m / 2} u\right|^{2}+\left|A^{m / 2} v\right|^{2}\right)+\left(|p|^{2}+|q|^{2}\right)+(u, p)+(v, q) .
\end{gathered}
$$

Lemma 1 1) Assume $a=\min \left\{1, m_{0}\right\}$ and $b=\max \left\{1, m_{1}\right\}$, then the norm derived from $G(z)$ is equivalent to the norm on $E_{0}$. Namely, $\forall z=(u, p, v, q) \in E_{0}$,

$$
a\|z\|_{E_{0}}^{2} \leq G(z) \leq b\|z\|_{E_{0}}^{2} .
$$

2) Assume $\lambda_{N+1}^{m} \geq 1,\left(4 m^{*}+2 \beta-3\right) \lambda_{N+1}^{m} \geq 4$ and $d=\max \left\{m^{*}+\frac{\beta}{2}+\frac{1}{4 \lambda_{N+1}^{m}}, 2\right\}$, then the norm derived from $F(z)$ is equivalent to the norm on $E_{0}$. Namely, $\forall z=(u, p, v, q) \in Q_{N} E_{0}$,

$$
\frac{3}{4}\|z\|_{E_{0}}^{2} \leq F(z) \leq d\|z\|_{E_{0}}^{2} .
$$

Proof. 1) According to (H1), we can get the conclusion easily.

2) Due to $z \in Q_{N} E_{0}$, applying Holder and Young inequality, and noticing the result of the projection, we get

$$
\begin{aligned}
F(z) & =\left(m^{*}+\frac{\beta}{2}\right)\left(\left|A^{m / 2} u\right|^{2}+\left|A^{m / 2} v\right|^{2}\right)+\left(|p|^{2}+|q|^{2}\right)+(u, p)+(v, q) \\
& \geq\left(m^{*}+\frac{\beta}{2}-\frac{1}{\lambda_{N+1}^{m}}\right)\left(\left|A^{m / 2} u\right|^{2}+\left|A^{m / 2} v\right|^{2}\right)+\frac{3}{4}\left(|p|^{2}+|q|^{2}\right) \\
& \geq \frac{3}{4}\|z\|_{E_{0}}^{2} .
\end{aligned}
$$




$$
\begin{aligned}
F(z) & =\left(m^{*}+\frac{\beta}{2}\right)\left(\left|A^{m / 2} u\right|^{2}+\left|A^{m / 2} v\right|^{2}\right)+\left(|p|^{2}+|q|^{2}\right)+(u, p)+(v, q) \\
& \leq\left(m^{*}+\frac{\beta}{2}+\frac{1}{4 \lambda_{N+1}^{m}}\right)\left(\left|A^{m / 2} u\right|^{2}+\left|A^{m / 2} v\right|^{2}\right)+2\left(|p|^{2}+|q|^{2}\right) \\
& \leq d\|z\|_{E_{0}}^{2} .
\end{aligned}
$$

Lemma 1 is proved completely.

Lemma 2 Suppose (H1)-(H2) hold, $u, v$ and $\bar{u}, \bar{v}$ are two solutions of problem (1.1)- (1.5), let $k=b / a$, then we have

$$
\forall t \geq 0, \quad\|Z(t)\|_{E_{0}}^{2} \leq k e^{c t}\|Z(0)\|_{E_{0}}^{2} .
$$

Where $c$ is a constant depending only on the data $\left(m^{*}, C_{1}, C_{2}, C_{3}, K_{1}, K_{2}\right)$.

Proof. Set $\omega=u-\bar{u}, \omega_{t}=p-\bar{p}, \theta=v-\bar{v}, \theta_{t}=q-\bar{q}, Z=\left(\omega, \omega_{t}, \theta, \theta_{t}\right)$, since $\omega, \theta$ satisfy the following equations (3.6), that is

$$
\begin{aligned}
& \omega_{t t}+M(s)(-\Delta)^{m} u-M(\bar{s})(-\Delta)^{m} \bar{u}+\beta(-\Delta)^{m} \omega_{t}+g_{1}(u, v)-g_{1}(\bar{u}, \bar{v})=0, \\
& \theta_{t t}+M(s)(-\Delta)^{m} v-M(\bar{s})(-\Delta)^{m} \bar{v}+\beta(-\Delta)^{m} \theta_{t}+g_{2}(u, v)-g_{2}(\bar{u}, \bar{v})=0 .
\end{aligned}
$$

Taking the inner product of (3.6) with $\omega_{t}$ and $\theta_{t}$ respectively, and adding them, we obtain

$$
\begin{aligned}
& \frac{1}{2} \frac{d}{d t}\left(\left|\omega_{t}\right|^{2}+\left|\theta_{t}\right|^{2}\right)+\beta\left(\left|D^{m} \omega_{t}\right|^{2}+\left|D^{m} \theta_{t}\right|^{2}\right) \\
& \quad+\left(M(s)(-\Delta)^{m} u-M(\bar{s})(-\Delta)^{m} \bar{u}, \omega_{t}\right) \\
& \quad+\left(M(s)(-\Delta)^{m} v-M(\bar{s})(-\Delta)^{m} \bar{v}, \theta_{t}\right) \\
& \quad+\left(g_{1}(u, v)-g_{1}(\bar{u}, \bar{v}), \omega_{t}\right)+\left(g_{2}(u, v)-g_{2}(\bar{u}, \bar{v}), \theta_{t}\right)=0 .
\end{aligned}
$$

Further

$$
\begin{aligned}
& \left(M(s)(-\Delta)^{m} u-M(\bar{s})(-\Delta)^{m} \bar{u}, \omega_{t}\right) \\
= & \frac{M(s)}{2} \frac{d}{d t}\left|D^{m} \omega\right|^{2}+M^{\prime}(\xi)\left(\left(\left|D^{m} u\right|+\left|D^{m} \bar{u}\right|\right)\left|D^{m} \omega\right|+\left(\left|D^{m} v\right|+\left|D^{m} \bar{v}\right|\right)\left|D^{m} \theta\right|\right)\left|(-\Delta)^{m} \bar{u}\right|\left|\omega_{t}\right|,
\end{aligned}
$$

and

$$
\begin{aligned}
& M^{\prime}(\xi)\left(\left(\left|D^{m} u\right|+\left|D^{m} \bar{u}\right|\right)\left|D^{m} \omega\right|+\left(\left|D^{m} v\right|+\left|D^{m} \bar{v}\right|\right)\left|D^{m} \theta\right|\right)\left|(-\Delta)^{m} \bar{u}\right|\left|\omega_{t}\right| \\
\leq & C_{1}\left(\left|D^{m} \omega\right|^{2}+\left|D^{m} \theta\right|^{2}+\frac{\left|\omega_{t}\right|^{2}}{2}\right),
\end{aligned}
$$

similarly

$$
\begin{aligned}
& \left(M(s)(-\Delta)^{m} v-M(\bar{s})(-\Delta)^{m} \bar{v}, \theta_{t}\right) \\
= & \frac{M(s)}{2} \frac{d}{d t}\left|D^{m} \theta\right|^{2}+M^{\prime}(\eta)\left(\left(\left|D^{m} u\right|+\left|D^{m} \bar{u}\right|\right)\left|D^{m} \omega\right|+\left(\left|D^{m} v\right|+\left|D^{m} \bar{v}\right|\right)\left|D^{m} \theta\right|\right)\left|(-\Delta)^{m} \bar{v}\right|\left|\theta_{t}\right|,
\end{aligned}
$$

and

$$
\begin{aligned}
& M^{\prime}(\eta)\left(\left(\left|D^{m} u\right|+\left|D^{m} \bar{u}\right|\right)\left|D^{m} \omega\right|+\left(\left|D^{m} v\right|+\left|D^{m} \bar{v}\right|\right)\left|D^{m} \theta\right|\right)\left|(-\Delta)^{m} \bar{v}\right|\left|\theta_{t}\right| \\
\leq & C_{2}\left(\left|D^{m} \omega\right|^{2}+\left|D^{m} \theta\right|^{2}+\frac{\left|\theta_{t}\right|^{2}}{2}\right) .
\end{aligned}
$$


Then by the assumption (H2), there exist constants $K_{1}, K_{2}$, such that $\left|g_{i u}\right| \leq K_{i},\left|g_{i v}\right| \leq K_{i}$, for $(i=1,2)$, we get

$$
\begin{aligned}
& \left|\left(g_{1}(u, v)-g_{1}(\bar{u}, \bar{v}), \omega_{t}\right)\right| \leq \int_{\Omega}\left|g_{1}(u, v)-g_{1}(\bar{u}, \bar{v})\right|\left|\omega_{t}\right| d x \\
= & \int_{\Omega} \mid g_{1 u}\left(\bar{u}+\theta_{1}(u-\bar{u}), \bar{v}+\theta_{1}(v-\bar{v})\right)(u-\bar{u})+ \\
& g_{1 v}\left(\bar{u}+\theta_{1}(u-\bar{u}), \bar{v}+\theta_{1}(v-\bar{v})\right)(v-\bar{v})|| \omega_{t} \mid d x \\
\leq & K_{1}\left(|\omega|\left|\omega_{t}\right|+|\theta|\left|\omega_{t}\right|\right) \leq K_{1}\left(\lambda_{1}^{-m}\left|D^{m} \omega\right|^{2}+\lambda_{1}^{-m}\left|D^{m} \theta\right|^{2}+\frac{\left|\omega_{t}\right|^{2}}{2}\right),
\end{aligned}
$$

similarly

$$
\begin{aligned}
& \left|\left(g_{2}(u, v)-g_{2}(\bar{u}, \bar{v}), \theta_{t}\right)\right| \leq \int_{\Omega}\left|g_{2}(u, v)-g_{2}(\bar{u}, \bar{v})\right|\left|\theta_{t}\right| d x \\
= & \int_{\Omega} \mid g_{2 u}\left(\bar{u}+\theta_{2}(u-\bar{u}), \bar{v}+\theta_{2}(v-\bar{v})\right)(u-\bar{u})+ \\
& g_{2 v}\left(\bar{u}+\theta_{2}(u-\bar{u}), \bar{v}+\theta_{2}(v-\bar{v})\right)(v-\bar{v})|| \theta_{t} \mid d x \\
\leq & K_{2}\left(\lambda_{1}^{-m}\left|D^{m} \omega\right|^{2}+\lambda_{1}^{-m}\left|D^{m} \theta\right|^{2}+\frac{\left|\theta_{t}\right|^{2}}{2}\right) .
\end{aligned}
$$

From above, we can conduct

$$
\begin{aligned}
& \frac{d}{d t}\left\{m^{*}\left(\left|D^{m} \omega\right|^{2}+\left|D^{m} \theta\right|^{2}\right)+\left(\left|\omega_{t}\right|^{2}+\left|\theta_{t}\right|^{2}\right)\right\}+2 \beta\left(\left|D^{m} \omega_{t}\right|^{2}+\left|D^{m} \theta_{t}\right|^{2}\right) \\
\leq & \left(2 C_{1}+2 C_{2}+2 K_{1} \lambda_{1}^{-m}+2 K_{2} \lambda_{1}^{-m}\right)\left(\left|D^{m} \omega\right|^{2}+\left|D^{m} \theta\right|^{2}\right)+ \\
& \left(C_{1}+K_{1}\right)\left|\omega_{t}\right|^{2}+\left(C_{2}+K_{2}\right)\left|\theta_{t}\right|^{2},
\end{aligned}
$$

here, let

$$
C_{3}=2\left(C_{1}+C_{2}\right)+2 \lambda_{1}^{-m}\left(K_{1}+K_{2}\right), c=\max \left\{C_{3} / m^{*}, C_{1}+K_{1}, C_{2}+K_{2}\right\},
$$

so

$$
\frac{d}{d t}\left\{m^{*}\left(\left|D^{m} \omega\right|^{2}+\left|D^{m} \theta\right|^{2}\right)+\left(\left|\omega_{t}\right|^{2}+\left|\theta_{t}\right|^{2}\right)\right\} \leq c\left\{m^{*}\left(\left|D^{m} \omega\right|^{2}+\left|D^{m} \theta\right|^{2}\right)+\left(\left|\omega_{t}\right|^{2}+\left|\theta_{t}\right|^{2}\right)\right\} .
$$

By formula (3.1)

$$
\frac{d}{d t} G(Z(t)) \leq c G(Z(t))
$$

Using Gronwall inequality

$$
G(Z(t)) \leq e^{c t} G(Z(0))
$$

Further, by (3.3) the equivalence of norm

$$
\|Z(t)\|_{E_{0}}^{2} \leq k e^{c t}\|Z(0)\|_{E_{0}}^{2}
$$

Lemma 2 is proved completely.

Lemma 3 Suppose (H1)-(H2) hold, $u, v$ and $\bar{u}, \bar{v}$ are two solutions of problem (1.1)- (1.5), let $N$ be such that $(\varphi, \psi)=q_{N}(\omega, \theta)$ and $Q=\left(\varphi, \varphi_{t}, \psi, \psi_{t}\right) \in Q_{N} Z$, then we have

$$
\frac{d}{d t} F(Q(t))+\frac{\mathcal{E}_{*}}{4} F(Q(t)) \leq \frac{C_{*}}{\varepsilon_{*} \lambda_{N+1}^{m}}\|Z(t)\|_{E_{0}}^{2} .
$$


Where $\varepsilon_{*}=\min \left\{\frac{m_{0}}{m^{*}+\beta / 2}, 2 \beta \lambda_{N+1}^{m}-1\right\} \in(0,1)$, and $C_{*}$ is a constant depending only on the data $\left(C_{4}, R_{1}, K\right)$.

Proof. Apply $q_{N}$ to (3.6), we get

$$
\begin{aligned}
& \varphi_{t t}+\beta(-\Delta)^{m} \varphi_{t}=-q_{N}\left(M(s)(-\Delta)^{m} u-M(\bar{s})(-\Delta)^{m} \bar{u}\right)-q_{N}\left(g_{1}(u, v)-g_{1}(\bar{u}, \bar{v})\right), \\
& \psi_{t t}+\beta(-\Delta)^{m} \psi_{t}=-q_{N}\left(M(s)(-\Delta)^{m} v-M(\bar{s})(-\Delta)^{m} \bar{v}\right)-q_{N}\left(g_{2}(u, v)-g_{2}(\bar{u}, \bar{v})\right) .
\end{aligned}
$$

Multiplying (3.9) by $2 \varphi_{t}, \varphi$ and $2 \psi_{t}, \psi$ respectively and integrating over $\Omega$, we have

$$
\begin{aligned}
& \frac{d}{d t}\left\{\left(m^{*}+\frac{\beta}{2}\right)\left(\left|D^{m} \varphi\right|^{2}+\left|D^{m} \psi\right|^{2}\right)+\left(\left|\varphi_{t}\right|^{2}+\left|\psi_{t}\right|^{2}\right)+\left(\varphi_{t}, \varphi\right)+\left(\psi_{t}, \psi\right)\right\}+ \\
& m_{0}\left(\left|D^{m} \varphi\right|^{2}+\left|D^{m} \psi\right|^{2}\right)+\left(2 \beta \lambda_{N+1}^{m}-1\right)\left(\left|\varphi_{t}\right|^{2}+\left|\psi_{t}\right|^{2}\right)+\varepsilon_{*}\left(\left(\varphi_{t}, \varphi\right)+\left(\psi_{t}, \psi\right)\right) \\
\leq & \varepsilon_{*}\left(\left(\varphi_{t}, \varphi\right)+\left(\psi_{t}, \psi\right)\right)-\left\{q_{N}(M(s)-M(\bar{s}))(-\Delta)^{m} \bar{u}, 2 \varphi_{t}+\varphi\right\}- \\
& \left\{q_{N}(M(s)-M(\bar{s}))(-\Delta)^{m} \bar{v}, 2 \psi_{t}+\psi\right\}- \\
& \left\{q_{N}\left(g_{1}(u, v)-g_{1}(\bar{u}, \bar{v})\right), 2 \varphi_{t}+\varphi\right\}-\left\{q_{N}\left(g_{2}(u, v)-g_{2}(\bar{u}, \bar{v})\right), 2 \psi_{t}+\psi\right\} \\
= & \varepsilon_{*}\left(\left(\varphi_{t}, \varphi\right)+\left(\psi_{t}, \psi\right)\right)-\left(\Gamma_{1}, 2 \varphi_{t}+\varphi\right)-\left(\Gamma_{2}, 2 \psi_{t}+\psi\right)- \\
& \left(\Gamma_{3}, 2 \varphi_{t}+\varphi\right)-\left(\Gamma_{4}, 2 \psi_{t}+\psi\right) .
\end{aligned}
$$

Owing to (H1), we estimate

$$
\begin{aligned}
\left|\Gamma_{1}\right| & \leq M^{\prime}(\xi)\left\{\left(\left|D^{m} u\right|+\left|D^{m} \bar{u}\right|\right)\left|D^{m} \omega\right|\left|(-\Delta)^{m} \bar{u}\right|_{\infty}+\left(\left|D^{m} v\right|+\left|D^{m} \bar{v}\right|\right)\left|D^{m} \theta\right|\left|(-\Delta)^{m} \bar{u}\right|_{\infty}\right\} \\
& \leq \frac{C_{4}}{\lambda_{N+1}^{m / 2}}\left\{\left(\left|\Delta^{m} u\right|+\left|\Delta^{m} \bar{u}\right|\right)\left|\Delta^{m} \bar{u}\right|\left|D^{m} \omega\right|+\left(\left|D^{m} v\right|+\left|D^{m} \bar{v}\right|\right)\left|\Delta^{m} \bar{u}\right|\left|D^{m} \theta\right|\right\} \\
& \leq \frac{2 C_{4} R_{1}^{2}}{\lambda_{N+1}^{m / 2}}\left(\left|D^{m} \omega\right|+\left|D^{m} \theta\right|\right),
\end{aligned}
$$

analogously

$$
\left|\Gamma_{2}\right| \leq \frac{2 C_{4} R_{1}^{2}}{\lambda_{N+1}^{m / 2}}\left(\left|D^{m} \omega\right|+\left|D^{m} \theta\right|\right)
$$

Further

$$
\begin{gathered}
\left|\Gamma_{3}\right| \leq \mid g_{1 u}\left(\bar{u}+\theta_{1}(u-\bar{u}), \bar{v}+\theta_{1}(v-\bar{v})\right)(u-\bar{u})+ \\
g_{1 v}\left(\bar{u}+\theta_{1}(u-\bar{u}), \bar{v}+\theta_{1}(v-\bar{v})\right)(v-\bar{v}) \mid \\
\leq K_{1}(|\omega|+|\theta|) \leq \frac{K_{1}}{\lambda_{N+1}^{m / 2}}\left(\left|D^{m} \omega\right|+\left|D^{m} \theta\right|\right),
\end{gathered}
$$

analogously

$$
\left|\Gamma_{4}\right| \leq K_{2}(|\omega|+|\theta|) \leq \frac{K_{2}}{\lambda_{N+1}^{m / 2}}\left(\left|D^{m} \omega\right|+\left|D^{m} \theta\right|\right) .
$$

So, the right side of (3.10) is estimated from the above 


$$
\begin{aligned}
& \varepsilon_{*}\left(\left|\varphi_{t}\right||\varphi|+\left|\psi_{t}\right||\psi|\right)+\frac{2 C_{4} R_{1}^{2}+K_{1}}{\lambda_{N+1}^{m / 2}}\left(\left|D^{m} \omega\right|+\left|D^{m} \theta\right|\right)\left(2\left|\varphi_{t}\right|+|\varphi|\right) \\
& +\frac{2 C_{4} R_{1}^{2}+K_{2}}{\lambda_{N+1}^{m / 2}}\left(\left|D^{m} \omega\right|+\left|D^{m} \theta\right|\right)\left(2\left|\psi_{t}\right|+|\psi|\right) \\
\leq & \frac{\varepsilon_{*}}{2}\left(\left|\varphi_{t}\right|^{2}+\left|\psi_{t}\right|^{2}\right)+\frac{\varepsilon_{*}}{2 \lambda_{N+1}^{m}}\left(\left|D^{m} \varphi\right|^{2}+\left|D^{m} \psi\right|^{2}\right)+ \\
& \frac{32\left(2 C_{4} R_{1}^{2}+K_{1}\right)^{2}}{\varepsilon_{*} \lambda_{N+1}^{m}}\left(\left|D^{m} \omega\right|^{2}+\left|D^{m} \theta\right|^{2}\right)+\frac{\varepsilon_{*}}{16}\left|\varphi_{t}\right|^{2}+ \\
& \frac{8\left(2 C_{4} R_{1}^{2}+K_{1}\right)^{2}}{\varepsilon_{*} \lambda_{N+1}^{m}}\left(\left|D^{m} \omega\right|^{2}+\left|D^{m} \theta\right|^{2}\right)+\frac{\varepsilon_{*}}{16 \lambda_{N+1}^{m}}\left|D^{m} \varphi\right|^{2}+ \\
& \frac{32\left(2 C_{4} R_{1}^{2}+K_{2}\right)^{2}}{\varepsilon_{*} \lambda_{N+1}^{m}}\left(\left|D^{m} \omega\right|^{2}+\left|D^{m} \theta\right|^{2}\right)+\frac{\varepsilon_{*}}{16}\left|\psi_{t}\right|^{2}+ \\
& \frac{8\left(2 C_{4} R_{1}^{2}+K_{2}\right)^{2}}{\varepsilon_{*} \lambda_{N+1}^{m}}\left(\left|D^{m} \omega\right|^{2}+\left|D^{m} \theta\right|^{2}\right)+\frac{\varepsilon_{*}}{16 \lambda_{N+1}^{m}}\left|D^{m} \psi\right|^{2} \\
\leq & \frac{3 \varepsilon_{*}}{4}\left\{\frac{3}{4 \lambda_{N+1}^{m}}\left(\left|D^{m} \varphi\right|^{2}+\left|D^{m} \psi\right|^{2}\right)+\frac{3}{4}\left(\left|\varphi_{t}\right|^{2}+\left|\psi_{t}\right|^{2}\right)\right\}+\frac{C_{*}}{\varepsilon_{*} \lambda_{N+1}^{m}}\left(\left|D^{m} \omega\right|^{2}+\left|D^{m} \theta\right|^{2}\right) .
\end{aligned}
$$

Where $C_{*}=40\left(2 C_{4} R_{1}^{2}+K\right)^{2}, K=\max \left\{K_{1}, K_{2}\right\}, \quad R_{1}$ is related to Theorem 2.2 of (Lin \& Hu, 2017).

Bring (3.11) into (3.10), we obtain

$$
\frac{d}{d t} F(Q(t))+\frac{\varepsilon_{*}}{4} F(Q(t)) \leq \frac{C_{*}}{\varepsilon_{*} \lambda_{N+1}^{m}}\|Z(t)\|_{E_{0}}^{2} .
$$

Proof of Lemma 3 is accomplished.

Lemma 4 There exists $C>0$ such that $\sup _{z_{0} \in B}\left\|z_{t}(t)\right\|_{E_{0}} \leq C, \forall t \geq 0$.

Proof. Differentiating equations (1.1)-(1.2) with respect to time $t$, we have

$$
\begin{aligned}
u_{t t t} & +M(s)(-\Delta)^{m} u_{t}+2 M^{\prime}(s)\left(\left(D^{m} u, D^{m} u_{t}\right)+\left(D^{m} v, D^{m} v_{t}\right)\right)(-\Delta)^{m} u \\
& +\beta(-\Delta)^{m} u_{t t}+g_{1 u}(u, v) u_{t}+g_{1 v}(u, v) v_{t}=0, \\
v_{t t t} & +M(s)(-\Delta)^{m} v_{t}+2 M^{\prime}(s)\left(\left(D^{m} u, D^{m} u_{t}\right)+\left(D^{m} v, D^{m} v_{t}\right)\right)(-\Delta)^{m} v \\
& +\beta(-\Delta)^{m} v_{t t}+g_{2 u}(u, v) u_{t}+g_{2 v}(u, v) v_{t}=0 .
\end{aligned}
$$

Taking the inner product of (3.13) with $u_{t t}$ and $v_{t t}$ respectively, we obtain

$$
\begin{aligned}
& \frac{d}{d t}\left\{M(s)\left(\left|D^{m} u_{t}\right|^{2}+\left|D^{m} v_{t}\right|^{2}\right)+\left|u_{t t}\right|^{2}+\left|v_{t t}\right|^{2}\right\}+2 \beta\left(\left|D^{m} u_{t t}\right|^{2}+\left|D^{m} v_{t t}\right|^{2}\right) \\
= & 2 M^{\prime}(s)\left(\left(D^{m} u, D^{m} u_{t}\right)+\left(D^{m} v, D^{m} v_{t}\right)\right) \\
& \left\{\left(\left|D^{m} u_{t}\right|^{2}+\left|D^{m} v_{t}\right|^{2}\right)-2\left(\left((-\Delta)^{m} u, u_{t t}\right)+\left((-\Delta)^{m} v, v_{t t}\right)\right)\right\}- \\
& \left\{\left(g_{1 u}(u, v) u_{t}, u_{t t}\right)+\left(g_{1 v}(u, v) v_{t}, u_{t t}\right)\right\}-\left\{\left(g_{2 u}(u, v) u_{t}, v_{t t}\right)+\left(g_{2 v}(u, v) v_{t}, v_{t t}\right)\right\} .
\end{aligned}
$$

From Theorem 2.1 and Theorem 2.2 of (Lin \& Hu, 2017), we know, there exist $c\left(R_{0}, R_{1}\right)>0$ and $\varepsilon>0$ suitably small,

$$
\begin{aligned}
& \frac{d}{d t}\left\{M(s)\left(\left|D^{m} u_{t}\right|^{2}+\left|D^{m} v_{t}\right|^{2}\right)+\left|u_{t t}\right|^{2}+\left|v_{t t}\right|^{2}\right\} \\
& \quad+(2-\varepsilon)\left\{M(s)\left(\left|D^{m} u_{t}\right|^{2}+\left|D^{m} v_{t}\right|^{2}\right)+\left|u_{t t}\right|^{2}+\left|v_{t t}\right|^{2}\right\} \leq c\left(R_{0}, R_{1}\right),
\end{aligned}
$$


therefore, Lemma 4 is certified by Gronwall inequality.

Lemma 5 For $\forall T>0$, the map $(t, z) \mapsto S(t) z$ is Lipschitz continuous on $[0, T] \times B$.

Proof. For $z_{0}, z_{1} \in B, t_{0}, t_{1} \in[0, T]$,

$$
\left\|S\left(t_{0}\right) z_{0}-S\left(t_{1}\right) z_{1}\right\|_{E_{0}} \leq\left\|S\left(t_{0}\right) z_{0}-S\left(t_{0}\right) z_{1}\right\|_{E_{0}}+\left\|S\left(t_{0}\right) z_{1}-S\left(t_{1}\right) z_{1}\right\|_{E_{0}} .
$$

The first term on the right side of the above formula is easily handled by Lemma 2, for the second item, by virtue of Lemma 4, we obtain

$$
\left\|S\left(t_{0}\right) z_{1}-S\left(t_{1}\right) z_{1}\right\|_{E_{0}}=\left\|z_{1}\left(t_{0}\right)-z_{1}\left(t_{1}\right)\right\|_{E_{0}} \leq\left|\int_{t_{0}}^{t_{1}}\left\|z_{1 t}(\tau)\right\|_{E_{0}} d \tau\right| \leq C\left|t_{0}-t_{1}\right|
$$

So

$$
\left\|S\left(t_{0}\right) z_{0}-S\left(t_{1}\right) z_{1}\right\|_{E_{0}} \leq L\left\{\left|t_{0}-t_{1}\right|+\left\|z_{0}-z_{1}\right\|_{E_{0}}\right\}
$$

it is set up for $L=L(T) \geq 0$.

Proof of Lemma 5 is completed.

Theorem 1 Select $t_{*}$, and satisfy

$$
2 d e^{-\varepsilon_{*} t_{*} / 4} \leq 1 / 128,
$$

and

$$
\frac{8 k C_{*}}{\varepsilon_{*}^{2} \lambda_{N+1}^{m}} e^{c t_{*}} \leq \frac{1}{128}
$$

then the solution operator $\{S(t)\}_{t>0}$ on the bounded subset $B$ in $E_{0}$ satisfies Lipschitz continuity and discrete squeezing property. If

$$
\left\|P_{N}\left(Z\left(t_{*}\right)\right)\right\|_{E_{0}} \leq\left\|Q_{N}\left(Z\left(t_{*}\right)\right)\right\|_{E_{0}},
$$

then

$$
\left\|Z\left(t_{*}\right)\right\|_{E_{0}}^{2} \leq \frac{1}{64}\|Z(0)\|_{E_{0}}^{2}
$$

Where $Z(t)=S(t) U-S(t) \bar{U}, U=\left(u_{0}, u_{1}, v_{0}, v_{1}\right), \bar{U}=\left(\bar{u}_{0}, \bar{u}_{1}, \bar{v}_{0}, \bar{v}_{1}\right)$.

Proof. By Lemma 2 and Lemma 3, we get

$$
\frac{d}{d t} F(Q(t))+\frac{\varepsilon_{*}}{4} F(Q(t)) \leq \frac{C_{*}}{\varepsilon_{*} \lambda_{N+1}^{m}}\|Z(t)\|_{E_{0}}^{2} \leq \frac{k C_{*}}{\varepsilon_{*} \lambda_{N+1}^{m}} e^{c t}\|Z(0)\|_{E_{0}}^{2} .
$$

By Gronwall inequality and Lemma 12 ), the following holds true

$$
\begin{gathered}
F(Q(t)) \leq F(Q(0)) e^{-\varepsilon_{*} t / 4}+\frac{4 k C_{*}}{\varepsilon_{*}^{2} \lambda_{N+1}^{m}} e^{c t}\|Z(0)\|_{E_{0}}^{2}, \\
\left\|Q_{N} Z(t)\right\|_{E_{0}}^{2} \leq\left(d e^{-\varepsilon_{*} t / 4}+\frac{4 k C_{*}}{\varepsilon_{*}^{2} \lambda_{N+1}^{m}} e^{c t}\right)\|Z(0)\|_{E_{0}}^{2} .
\end{gathered}
$$

From (3.15), (3.16), and $N$ so large, 


$$
\begin{aligned}
\left\|Z\left(t_{*}\right)\right\|_{E_{0}}^{2} & =\left\|P_{N} Z\left(t_{*}\right)\right\|_{E_{0}}^{2}+\left\|Q_{N} Z\left(t_{*}\right)\right\|_{E_{0}}^{2} \leq 2\left\|Q_{N} Z\left(t_{*}\right)\right\|_{E_{0}}^{2} \\
& \leq\left(2 d e^{-\varepsilon_{*}, t_{*} / 4}+\frac{8 k C_{*}}{\varepsilon_{*}^{2} \lambda_{N+1}^{m}} e^{c t_{*}}\right)\|Z(0)\|_{E_{0}}^{2} \leq \frac{1}{64}\|Z(0)\|_{E_{0}}^{2} .
\end{aligned}
$$

That is to say

$$
\left\|Z\left(t_{*}\right)\right\| \leq \frac{1}{8}\|Z(0)\|_{E_{0}} .
$$

Proof of Theorem 1 is completed.

\section{Conclusions}

In this paper, we study a class of high-order Kirchhoff-type equations. By using the method proposed by Eden et al. and combining our assumptions given in advance, we obtain the Lipschitz property and the discrete squeezing property, which prove its the existence of exponential attractor. Among them, we have maximum and minimum values in the bounded closed region according to the continuous function of mathematical analysis, we have made a limitation on $M(s)$, which needs further improvement. In later studies, I hope that I can deeply explore its greater possibilities.

\section{Acknowledgements}

The authors express their sincere thanks to the anonymous referee for his/her careful reading of the paper, giving valuable suggestions and comments, which have greatly improved the presentation of this paper.

\section{References}

Brochet, D., Hilhorst, D., \& Novick-cohen, A. (1994). Finite-dimensional exponential attractor for a model for order-disorder and phase separation. Appl. Math. Lett, 7(3), 83-87. https://doi.org/10.1016/0893-9659(94)90118-X

Chen, L., Wang, W., \& Lin, G. G. (2016). Exponential attractors and inertial manifolds for the higher-order nonlinear Kirchhoff-type equation. International Journal of Modern Communication Technologies \& Research, 4(11), 6-12.

Eden, A., \& Kalantarov, V. (1996). Finite dimensional attractors for a class of semilinear wave equations. Turk. Jour. Math, 20(3), 425-450.

Eden, A., \& Rakotoson, J. M. (1994). Exponential attractors for a doubly nonlinear equation. Jour. Math. Anal. Appl, 185, 321-339. https://doi.org/10.1006/jmaa.1994.1251

Eden, A., Foias, C., \& Kalantarov, V. (1998). A remark on two constructions of exponential attractors for $\alpha$-contractions. Jour. Dynam. Diff. Eq, 10(1), 37-45. https://doi.org/10.1023/A:1022636328133

Eden, A., Foias, C., Nicolaenko, B., \& Temam, R. (1994). Exponential attractors for dissipative evolution equations. New York: Masson Paris Wiely.

Fan, X. M., \& Yang, H. (2010). Exponential attractor and its fractal dimension for a second order lattice dynamical system. Jour. Math. Anal. Appl, 367, 350-359. https://doi.org/10.1016/j.jmaa.2009.11.003

Gao, P. M., \& Ma, Q. Z. (2011). The exponential attractor for Kuramoto-Sivashinsky Equation. Journal of Southwest University, 33(9), 25-28.

Han, L. J., \& Ma, Q. Z. (2011). The exponential attractor for the dissipative MKdV equation. Acta Analysis Functionalis Applicata, 13(1), 78-84.

Jia, L., \& Ma, Q. Z. (2017). The exponential attractor for nonlinear stretchable beam equation. Journal of Jilin University, 55(4), 839-844.

Kang, Y. H., \& Ma, Q. Z. (2012). The existence of exponential attractor for nonlinear Reaction-diffusion equation with derivative term. Jour. Syst. Sci.\& Math. Sci. Chin. Seri, 32(11), 1407-1412.

Li, K., \& Yang, Z. J. (2013). Exponential attractors for the strongly damped wave equation. Appl. Math. Comp, 220, 155-165. https://doi.org/10.1016/j.amc.2013.05.044

Lin, G. G., \& Hu, L. J. (2017). The gloabal attractor for a class of higher-order coupled Kirchhoff-type equations with strong linear damping. European Journal of Mathematics and Computer Science, 4(1), 63-77.

Lin, G. G., Lv, P. H., \& Lou, R. J. (2017). Exponential attractors and inertial manifolds for a class of nonlinear generalized Kirchhoff- Boussinesq model. Far East Journal of Mathematical Sciences, 101(9), 1913-1945.

https://doi.org/10.17654/MS101091913 
Ma, Q. Z., \& Liu, Y. F. (2011). The exponential attractors for a nonclassical diffusion equation with arbitrary polynomial growth. Chinese Journal of Engineering Mathematics, 28(1), 134-138.

Milani, A. (1992). Exponential attractors for semilinear wave equations with damping. Partial Differential Equations Banach Center Publications, 27, 327-335. https://doi.org/10.4064/-27-2-327-335

Shang, Y. D., \& Guo, B. L. (2005). Exponential attractor for the generalized symmetric regularized long wave equation with damping term. Applied Mathematics and Mechanics, 26(3), 283-291. https://doi.org/10.1007/BF02440077

Wang, S. P., Ma, Q. Z., \& Shao, X. K. (2011). The exponential attractor for the beam equation. Journal of Southwest University, 33(9), 29-35.

Wang, W. T., \& Ma, Q. Z. (2016). The existence of exponential attractor for suspension bridge equation. Acta Analysis Functionalis Applicata, 18(2), 212-219.

Yang, M. H., \& Sun, C. Y. (2010). Exponential attractors for the strongly damped wave equations. Nonl. Anal: Real World Applications, 11, 913-919. https://doi.org/10.1016/j.nonrwa.2009.01.022

Yang, Z. J., \& Liu, Z. M. (2015). Exponential attractor for the Kirchhoff equations with strong nonlinear damping and supercritical nonlinearity. Appl. Math. Lett, 46, 127-132. https://doi.org/10.1016/j.aml.2015.02.019

\section{Copyrights}

Copyright for this article is retained by the author(s), with first publication rights granted to the journal.

This is an open-access article distributed under the terms and conditions of the Creative Commons Attribution license (http://creativecommons.org/licenses/by/4.0/). 